\title{
Gradhiva
}

GRADHIV

Revue d'anthropologie et d'histoire des arts

$24 \mid 2016$

ARTchives

\section{Fragments d'histoire congolaise. Les archives coloniales réactivées du Mémorial Savorgnan de Brazza et de la Fresque de l'Afrique}

Fragments of Congolese history: The reactivated colonial archive of the Savorgnan de Brazza Memorial and of the Fresque de l'Afrique

\section{Nora Greani}

\section{(2) OpenEdition}

Journals

Édition électronique

URL : http://journals.openedition.org/gradhiva/3263

DOI : 10.4000/gradhiva.3263

ISSN : 1760-849X

Éditeur

Musée du quai Branly Jacques Chirac

Édition imprimée

Date de publication : 7 décembre 2016

Pagination : 82-105

ISBN : 978-2-35744-094-4

ISSN : 0764-8928

Référence électronique

Nora Greani, «Fragments d'histoire congolaise. Les archives coloniales réactivées du Mémorial

Savorgnan de Brazza et de la Fresque de l'Afrique », Gradhiva [En ligne], 24 | 2016, mis en ligne le 07 décembre 2019, consulté le 19 avril 2019. URL : http://journals.openedition.org/gradhiva/3263 ; DOI : 10.4000/gradhiva.3263 


\section{Fragments d'histoire congolaise}

Les archives coloniales réactivées du Mémorial Savorgnan de Brazza et de la Fresque de l'Afrique

par Nora Greani

En 2006, à Brazzaville, la décoration intérieure du Mémorial Savorgnan de Brazza est confiée à un collectif de peintres qui réalisent une vaste fresque, retraçant l'histoire du contact de l'explorateur avec la terre congolaise. Près de quarante ans plus tôt, la Fresque de l'Afrique avait été réalisée dans la capitale afin de commémorer la mise en place d'une République populaire socialiste. Dans ces deux œuvres officielles, on trouve une scène identique qui, bien qu'elle réactive la même archive coloniale, est investie de messages politiques antagonistes. Basé sur une analyse iconographique et sur le recueil de témoignages oraux, cet article étudie la fragmentation du récit historique sous le pinceau des artistes congolais. Son principal enjeu est d'éclairer l'évolution du rapport officiel au passé colonial en République du Congo au travers de différents usages des archives. 
1. D'autres controverses existent comme l'absence de statue à l'effigie du chef (Makoko) qui céda son territoire à la France, ou le fait que le mémorial ait été édifié près du port fluvial communément appelé «le Beach », où disparurent, en 1999, plusieurs dizaines voire centaines de personnes suspectées d'appartenir aux milices de l'opposition. Voir Yengo 2006.
2. Trois ans après l'indépendance obtenue en 1960, le président

Fulbert Youlou est chassé du pouvoir lors d'un soulèvement populaire. Son successeur, Alphonse Massamba-Débat, fonde un parti unique (le Mouvement national de la révolution, au pouvoir de 1964 à 1968) et instaure le socialisme comme doctrine officielle de l'État. Il est finalement évincé par Marien Ngouabi qui forme un nouveau parti unique (le Parti congolais du travail en place de 1969 à 1991) et une République populaire le 31 décembre 1968 (voir Bertrand 1975 et Bernault 1996). À partir de la brève présidence de Joachim Yhombi-Opango, suivie de son renversement par Denis Sassou N'Guesso en 1979 , le pays opère une transition vers le capitalisme. Le début des années 1990 signe la fin officielle du socialisme et inaugure une longue période de crise politique, sociale et économique. En effet, l'ère de la démocratisation réclamée lors de la Conférence nationale dégénère rapidement en une succession de guerres civiles jusqu'au début des années 2000.

3. Par exemple, le drapeau national, réalisé d'après une maquette du peintre ponténégrin Michel Hengo, représente une faucille et une houe en référence au modèle soviétique; la houe est toutefois censée constituer un outil plus spécifiquement congolais.

4. Ces artistes sont Michel Hengo, Émile Mokoko, André Ombala et Jean Itoua, ainsi qu'un superviseur italien surnommé Dégo.

\section{ci-contre}

fig. 1

Atelier Nadar, Portrait de Pierre Savorgnan de Brazza, v. 1880. Photo (C) Ministère de la Culture - Médiathèque du Patrimoine, Dist. RMNGrand Palais / Atelier de Nadar.
À la fin du $x^{x}{ }^{e}$ siècle, l'explorateur charismatique Pierre Savorgnan de Brazza permet à la France de prendre possession de l'actuel territoire de la République du Congo. En 2006, sa dépouille est transférée d'Alger à Brazzaville, au sous-sol d'un imposant monument circulaire de facture néoclassique, entouré d'un jardin verdoyant et devancé par sa statue en pied. La première pierre de ce mémorial a été posée en février 2005, en présence des présidents congolais, gabonais et français (respectivement Denis Sassou N'Guesso, feu Omar Bongo Ondimba et Jacques Chirac). Cet événement correspond, en France, à l'adoption controversée de la loi n 2005-158 portant sur les aspects "positifs» de la colonisation (Boilley 2005). L'inauguration officielle du monument, le 3 octobre 2006 - en référence au 3 octobre 1880, date à laquelle Savorgnan de Brazza arbore le pavillon français et fonde Brazzaville -, s'est déroulée sur fond de polémiques internationales liées au coût de construction élevé et à la revalorisation officielle de la mémoire de la colonisation ${ }^{1}$.

Plusieurs chercheurs ont analysé les enjeux sociopolitiques du rapatriement des restes mortuaires du colonisateur dans ce temple de verre et de marbre. Traitant principalement de la réception médiatique du monument (Atondi-Monmondji 2006), de son traitement littéraire (Martin-Granel 2010), ainsi que des processus de sacralisation au cœur des trafics de corps et d'ossements (Bernault 2010a, Bernault 2010b, Bazenguissa-Ganga 2014, Tonda 2010), leurs analyses apportent une contribution notable aux champs de l'anthropologie du politique et de l'imaginaire. Dans le prolongement de leurs travaux, je souhaite ici aborder une composante du mémorial encore non étudiée: sa décoration intérieure. Au rez-de-chaussée se déploie en effet une fresque de quinze mètres, dont le dispositif stylistique et narratif mérite d'être observé de près. Réalisée par un collectif de treize peintres de l'École de peinture de Poto-Poto, célèbre institution culturelle locale, l'œuvre représente, en les célébrant, différents épisodes de l'histoire du contact de Savorgnan de Brazza avec la terre congolaise. Chaque scène se base sur un récit et une iconographie d'époque provenant de divers fonds d'archives.

Située dans le centre-ville, une autre fresque historique datant de 1970 pose un regard bien plus sombre sur le passé colonial. En 1968, le pays indépendant depuis 1960 devient la première République populaire du continent africain ${ }^{2}$. L'orientation politique socialiste dès 1963 a pour conséquence l'apparition d'un répertoire iconographique et d'une esthétique voués à la glorification de l'idéologie révolutionnaire, constitués d'emprunts variés (réalisme soviétique, apparat des leaders cubains, peinture d'Eugène Delacroix, etc.) adaptés au contexte national ${ }^{3}$. La Fresque de l'Afrique offre un remarquable aperçu de cette esthétique socialiste. L'œuvre se présente sous la forme d'une immense arche recouverte de centaines de carreaux de céramique peints à la main. Créée par quatre peintres issus d'ateliers indépendants et supervisés par un artiste italien ${ }^{4}$, celle-ci reprend des «morceaux choisis» de l'histoire nationale, depuis la période précoloniale jusqu'à l'avènement du socialisme scientifique.

Dans les deux cas, des peintres au service de l'État ont endossé le rôle de passeurs de mémoire officielle en puisant dans une documentation diverse - parfois la même - pour composer leurs œuvres. L'assemblage d'images 


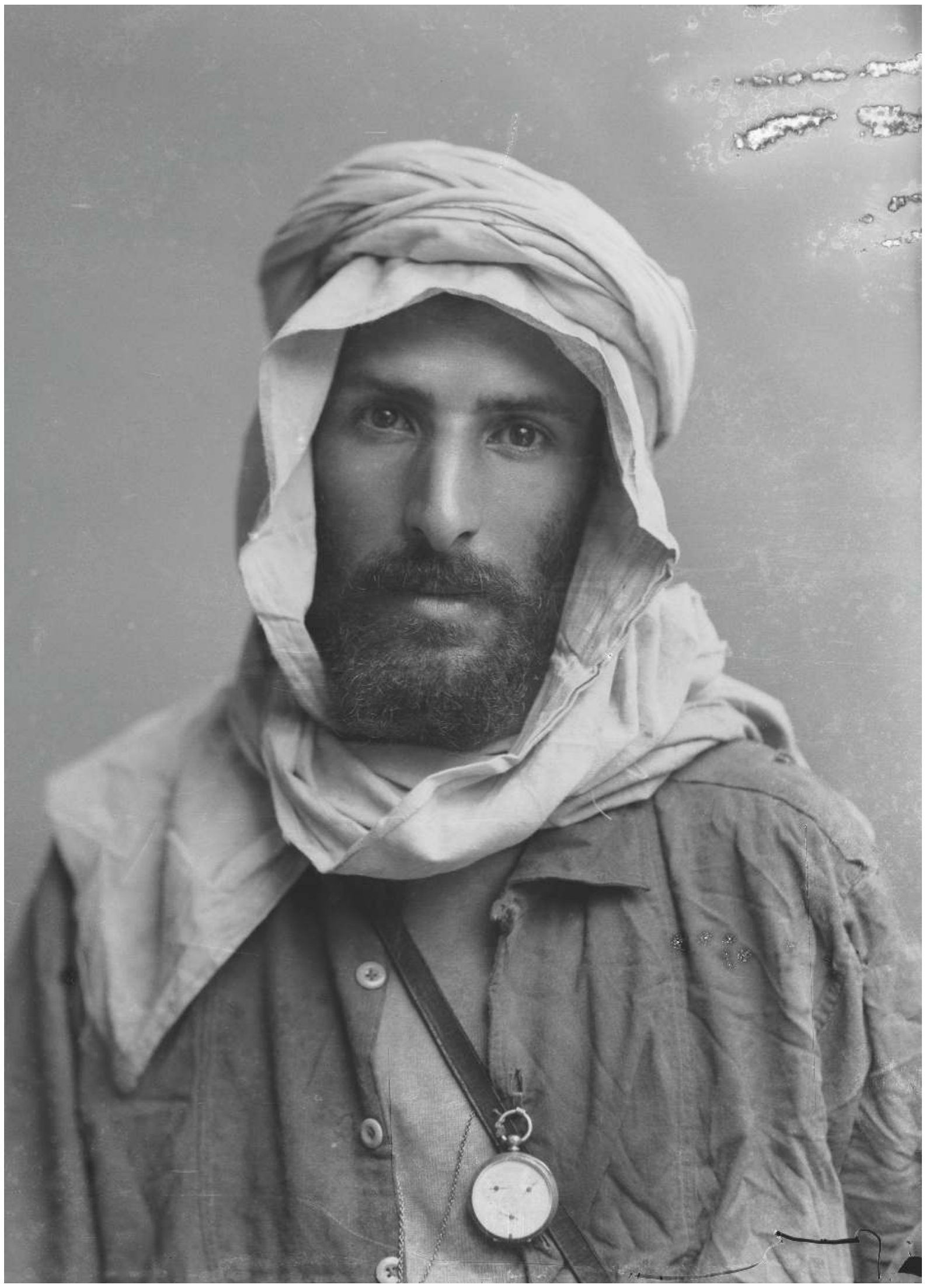




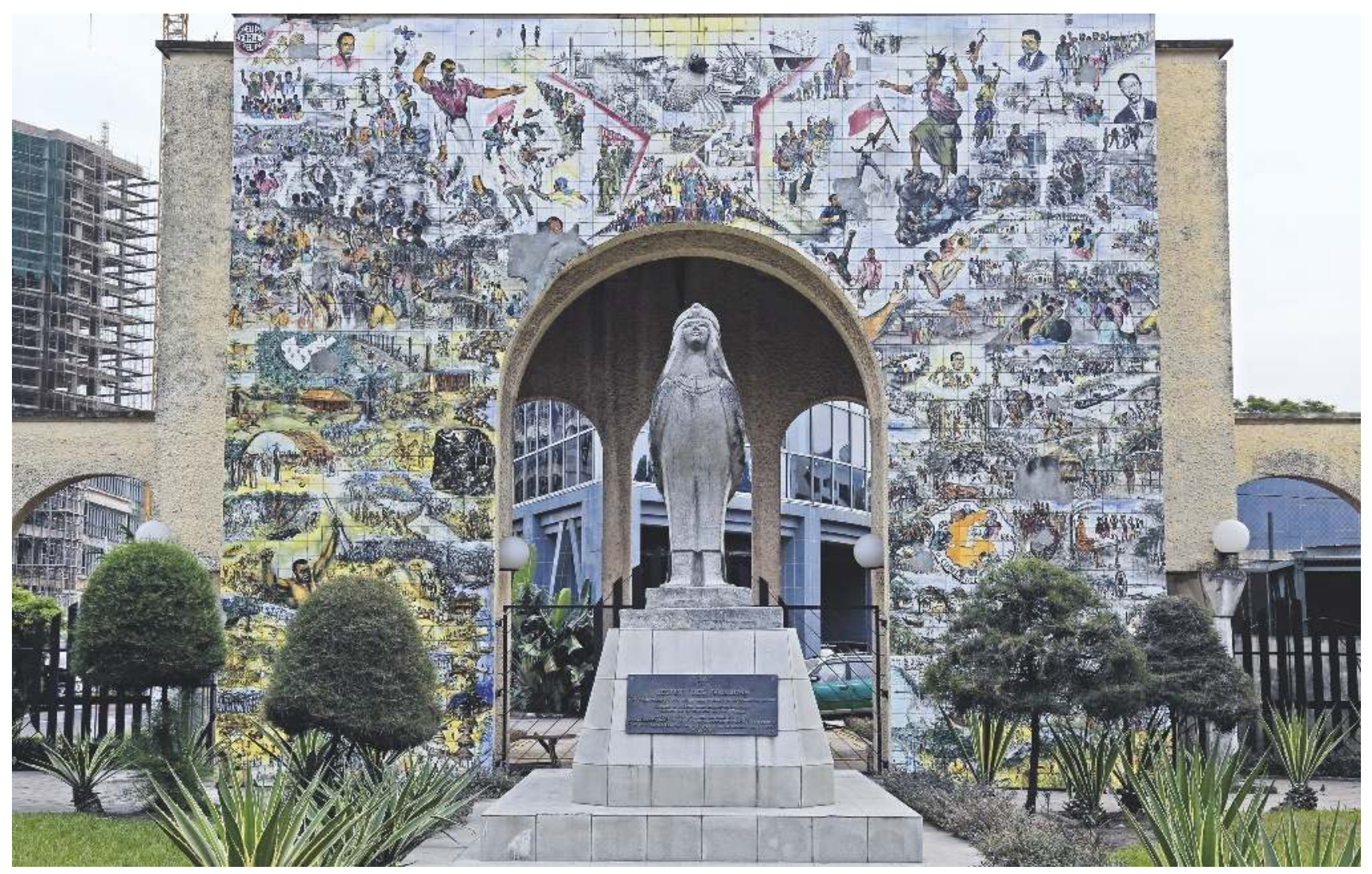

fig. 2

Baudouin Mouanda,

Fresque de l'Afrique,

Brazzaville, 2016

(c) Baudouin Mouanda. 
aboutit à deux mises en récit de l'histoire nationale, en accord avec l'idéologie politique dominante à une époque donnée, et témoigne des revirements idéologiques en République du Congo. En quoi les usages contrastés du matériau «archive» par les artistes éclairent-t-ils l'évolution du rapport à la colonisation? Plus largement, de quelle manière l'archive se donne-t-elle à voir dans des contextes géopolitiques où art et politique sont particulièrement intriqués?

Pour le comprendre, j'examinerai tout d'abord les pratiques et les réactions du public visé par les fresques puisque, comme le rappelle Nicos Hadjinicolaou, «aucune œuvre n'est jamais faite pour l'éternité ou pour un public futur [...] mais à partir du public et pour le public contemporain ". (Hadjinicolaou 1979: 3) Puis, j'analyserai comment, touche après touche, la fresque du mémorial procède à une réactivation non critique d'archives datant des premières heures de la colonisation. Cette réactivation prenant le contre-pied d'une tendance montante ces dernières années consistant pour les artistes contemporains d'Afrique à recycler de manière subversive les archives (Le Lay et al. [dir.] 2015; De Jong et Harney [dir.] 2015), je tenterai ensuite de cerner les raisons expliquant que les artistes de la fresque du mémorial aient choisi d'aller dans le sens de l'archive coloniale, ou «along the grain » (Stoler 2010). Enfin, je m'attarderai sur les enjeux de la représentation picturale d'une même archive, réalisée à près de quarante ans d'intervalle et traitée de manière quasi identique dans les deux fresques ${ }^{5}$.

\section{Les fresques vues "du bas ${ }^{6}$ "}

La Fresque de l'Afrique et celle du mémorial sont exposées de manière permanente et publique à Brazzaville selon des modalités qu'il convient de distinguer.

À l'origine, l'arche socialiste constituait l'entrée d'un marché couvert. Elle était donc intimement liée à la vie quotidienne des Congolais qui, en la franchissant, jouissaient du décor élaboré par les peintres, et donc, en creux, par le gouvernement, à leur intention. C'est d'ailleurs presque malgré eux que ceux-là profitaient du spectacle pictural offert, puisqu'ils venaient en ce lieu vendre ou acheter différents produits de consommation courante. En 1980, au moment du boom pétrolier, le marché fut démoli et remplacé par un immeuble moderne de onze étages, annulant du même coup la fonction première de l'œuvre. Une barrière restreint désormais le passage sous l'arche et une sculpture représentant un oiseau anthropomorphe, don du gouvernement égyptien, a été installée à l'avant de la fresque en 2006. Ces éléments qui en obstruent l'accès ont définitivement modifié la relation de l'œuvre à son public, et l'immense écran plat récemment accolé, diffusant des publicités ou les matchs de football de l'équipe nationale, a brouillé encore un peu plus le message communiste initial.

La fresque du mémorial, qui n'est quant à elle pas à l'air libre, ne bénéficie pas de la même visibilité. Décorant l'intérieur de l'imposant édifice, elle a été pensée dès l'origine pour interagir avec ses spectateurs de la même manière que dans un musée traditionnel. Pour comprendre cette situation, il convient de souligner que la République du Congo ne dispose plus de musée
5. Cet article se base sur des données de terrain recueillies entre 2009 et 2013 durant ma thèse de doctorat à l'École des hautes études en sciences sociales ainsi que sur un travail de recherche postdoctoral au sein du Laboratoire d'excellence Création, arts et patrimoines (Labex CAP) dans le cadre d'un rattachement aux laboratoires: IIAC-Lahic, musée du quai Branly et HiCSA entre 2014 et 2015. J'exprime ici ma reconnaissance à JeanLoup Amselle et à Daniel Fabre pour leur confiance et leurs conseils durant ces recherches.

6. Cette formulation fait référence à l'analyse du politique par le bas: Bayart 1981; Bayart et al. 2008 [1981]. Voir également «Grands hommes vus d'en bas. L'iconographie officielle et ses usages populaires", Julien Bonhomme et Nicolas Jaoul (dir.) Gradhiva 11, 2010. 
7. La construction du mémorial s'inscrit dans un nouveau contexte politico-idéologique propre à la situation post-guerre civile ainsi qu'à l'adoption du libéralisme économique et au rejet du socialisme scientifique. national depuis de nombreuses années. Le jeune État en a fait construire un cinq ans après son accession à l'indépendance, mais le musée a été détruit à la suite de l'assassinat du président Marien Ngouabi, le 18 mars 1977. La décision est alors prise de raser le musée ainsi que les bâtiments avoisinants afin de libérer l'espace nécessaire à l'implantation d'une «Place du sacrifice suprême " où est érigée une statue en pied du président socialiste défunt. La commémoration du martyr de la République populaire prime donc sur la sauvegarde de l'un des symboles forts de l'État-nation. Les collections sont transférées dans un modeste local du centre-ville inadapté à leur conservation et leur sécurité, sans espace dévolu à l'exposition des œuvres d'art. Plus de cinquante ans après l'indépendance, le pays ne dispose donc pas de musée national à l'intention des citoyens et des touristes, ni même des artistes locaux susceptibles d'y exposer leurs œuvres. Cette situation regrettable est moins le fruit du désintérêt de l'État que d'un nouveau bouleversement de l'ordre des priorités du champ politique, propre à la première décennie du $x^{e}{ }^{e}$ siècle $^{7}$ : dans l'échelle des urgences commémoratives, le patriote socialiste Ngouabi cède sa place au colonisateur et néanmoins «bienfaiteur » Savorgnan de Brazza, et plutôt qu'un musée national, c'est le coûteux mémorial qui est édifié dans la capitale.

En franchissant la porte du mémorial, le visiteur pénètre dans un espace (très) climatisé. Des haut-parleurs émettent des musiques en tous genres, qui vont des chants traditionnels au célèbre titre "Yamore» de Salif Keïta et Cesaria Evora, en passant par "Pour que tu m'aimes encore» de Céline Dion. Plusieurs écrans plats fixés en hauteur diffusent en continu des extraits du film de Léon Poirier, Brazza ou l'Épopée du Congo (1939), pur produit de propagande coloniale. Un portrait sous verre du président récemment réélu, Sassou N'Guesso, trône en hauteur. L'espace prend des allures de musée miniature avec ses masques et statuettes d'art premier installés sous d'élégantes cloches de verre. Un cordon rouge, habituellement présent dans les musées pour guider les pas des visiteurs, empêche ici de trop s'approcher de la fresque. II s'agit d'autant de signes manifestes, faute de mieux, de la symbolique muséale dans la capitale.

Le type de public visé pour chacune des deux fresques est lié au contexte politique et idéologique de leur apparition. Ainsi, du temps du régime du parti unique socialiste, l'État cherche à démocratiser autant que possible l'accès à la culture (théâtre, ballet, orchestre, etc.). Considéré comme un instrument de propagande - au même titre que les émissions de radio ou les articles de presse (Bonnafé 1968: 359) -, l'art visuel offre l'avantage de diffuser l'idéologie officielle sans exclure les populations analphabètes. Le Parti-État se charge donc, souvent à l'issue d'un concours public, de faire fabriquer des timbres (voués à une circulation nationale ou internationale) et des billets de faible valeur (manipulés par les couches les plus basses de la société) ou de faire décorer des lieux publics. Astucieusement placée à l'entrée d'un marché populaire, la fresque socialiste s'inscrit dans la même logique. La fresque du mémorial, quant à elle, est publique certes, à la condition d'obtenir des gardiens un droit de passage depuis le portail extérieur (ce qui suppose de présenter ses papiers d'identité), puis de traverser un jardin surveillé et d'entrer dans le bâtiment, lui aussi gardé. L'œuvre s'adresse à un public restreint, plutôt privilégié et expatrié, familier 


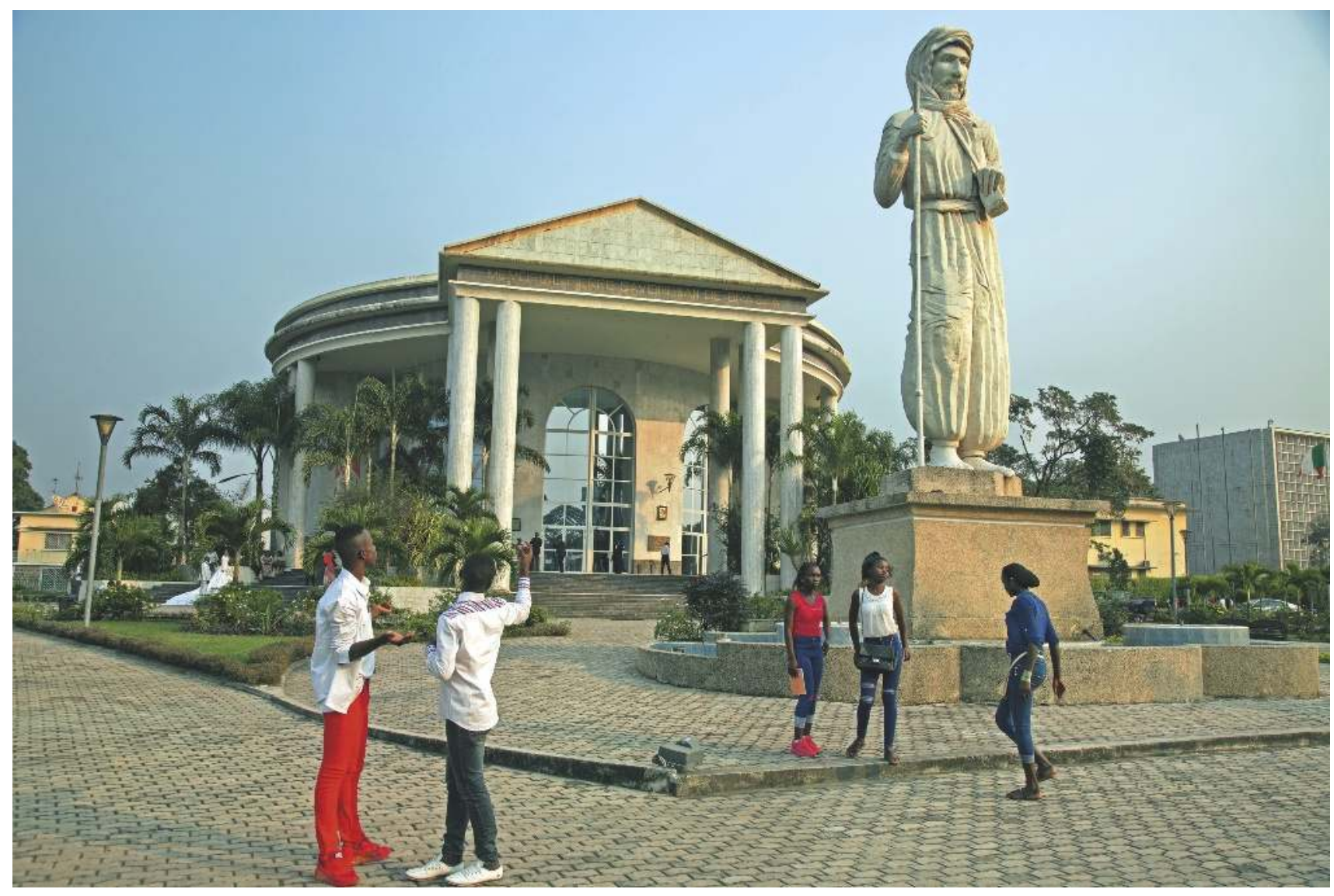

fig. 3

Baudouin Mouanda,

Mémorial Savorgnan de

Brazza, Brazzaville, 2016

(C) Baudouin Mouanda. 
8. Extraits de journaux de terrain entre 2009 et 2013 à Brazzaville.

9. Cette expression s'inspire de la notion de cascade des images théorisée par Bruno Latour qui désigne l'intertextualité pratiquée par les artistes dans leurs œuvres, c'est-à-dire la relation entretenue entre une image et toutes celles déjà produites (Latour 2009). de la pratique des musées. La visite ne se produit probablement qu'une seule fois, alors que l'emplacement de la Fresque de l'Afrique, à l'entrée du marché et sur une route très fréquentée de la capitale, en faisait une œuvre souvent regardée.

En enquêtant sur place, j'ai pu constater à quel point l'arche et le mémorial sont investis par une large partie de la population d'un imaginaire surnaturel. Des deux monuments émaneraient des forces néfastes directement imputables au président. En effet, on considère que, fétiches et magie noire à l'appui, Sassou N'Guesso utiliserait certains édifices publics pour accroître son prestige auprès de la population et neutraliser ses adversaires politiques. Ce type de croyance populaire appartient à la longue liste des soupçons dont celui-ci fait l'objet depuis sa prise de pouvoir (Gruénais et al. 1995; Tonda 2002). En ce qui concerne la Fresque de l'Afrique, c'est la sculpture d'inspiration égyptienne, élément étranger posé devant l'arche, qui est suspectée de diffuser des forces occultes, au point que certains Congolais préfèrent la contourner. Dans la même veine, de nombreuses personnes sont réticentes à entrer dans le mémorial. Certaines le considèrent comme un "cimetière" hanté par les morts (les caveaux de Savorgnan de Brazza et de sa famille sont installés au sous-sol), d'autres le soupçonnent d'être le siège de cultes maléfiques - en particulier de «démoniaques" cérémonies franc-maçonniques du président. De mes entretiens avec des mères de famille réalisés à la sortie des écoles maternelles et primaires de Brazzaville ressort la crainte de certaines d'entre elles de voir leurs enfants pénétrer dans ce lieu, en particulier lors de sorties scolaires. Voici quelques-uns des ordres intimés à leurs enfants à l'évocation du mémorial: "Toi là, si tu rentres là-bas, ils vont te bouffer!», « Même si on te demande d'y aller, c'est non!", "C'est un lieu satanique, tu n'y vas pas ${ }^{8}$ ", etc. Si le refus d'entrer dans le mémorial est principalement le fait de personnes modestes ayant un faible niveau d'études, il concerne également des personnes aisées et éduquées qui refusent de rendre hommage à un colonisateur, quel qu'il soit. Ainsi, certains intellectuels s'interdisent d'entrer dans ce qu'ils considèrent comme un "cimetière pour Blancs» et déclinent toute invitation à participer aux séminaires ou colloques régulièrement organisés sur place.

\section{Réactivations en cascade}

Chacun des quinze épisodes qui composent la fresque du mémorial provient d'une imagerie coloniale (croquis, peintures, gravures, etc.) issue de récits ou, plus rarement, de photographies d'époque. L'œuvre se caractérise donc par de successives artifications et ré-artifications (Heinich, Shapiro 2012) d'archives coloniales - c'est-à-dire le passage du «non art » (l'archive) à de l'art (art colonial puis art actuel). On parlera de réactivation d'archives "en cascade" pour qualifier le processus ayant abouti à l'ensemble de la réalisation 9 .

Pour comprendre les déplacements de frontières et l'intertextualité qui caractérisent cette fresque, revenons à la fin du xixe siècle. Savorgnan de Brazza mène trois missions exploratoires dans l'Ouest africain en tant qu'officier de la marine française. Entre 1875 et 1878, il remonte le cours du fleuve Ogooué (traversant l'actuel Gabon) à bord d'un bateau à vapeur, en vue d'ouvrir une nouvelle voie de communication vers l'Afrique centrale. Au cours 
de sa deuxième mission, de 1879 à 1882, il parvient à faire signer un traité de protectorat à llo ler, le Makoko (chef coutumier) des Batéké. Une fois ce traité ratifié par le Parlement français, Savorgnan de Brazza repart de 1883 à 1885 afin de remettre le document au Makoko, officialisant ainsi la cession de ses «droits héréditaires de suprématie ${ }^{\mathbf{1 0}}$ » territoriale à la France. Cette troisième mission se déroule dans un contexte tendu de compétition politique et de course à l'expansion territoriale en Europe ${ }^{\mathbf{1 1}}$, que cherche à apaiser la Conférence de Berlin, entre novembre 1884 et février 1885, en répartissant les territoires d'Afrique entre les puissances coloniales ${ }^{12}$.

Les explorations de Savorgnan de Brazza ont produit une quantité considérable de documents devenus archives: carnets de route, rapports de mission, correspondance officielle et privée, brouillons ou volumes d'articles de presse. Majoritairement conservées en France, ces archives font actuellement l'objet de modestes politiques de rapatriement au sein du mémorial. Ainsi, vingt-neuf documents ont été rachetés par le gouvernement congolais en $2015^{\mathbf{1 3}}$. Faisant écho au statut de musée national «au rabais " évoqué plus haut, il semble que le mémorial aspire également à devenir un centre d'archives dans une capitale où le bâtiment officiellement destiné à cet usage connaît de sérieuses difficultés de fonctionnement. Entre les deux désignations du monument, «mémorial » (son appellation officielle) ou «mausolée» (plus communément, pour rappeler sa fonction première: accueillir une sépulture), mon choix se porte donc sur le terme «mémorial» qui rend bien compte de ces multiples statuts (mausolée, musée, centre d'archives, etc.).

Outre les archives textuelles «classiques", il existe un flot d'images: photographies, affiches, illustrations, esquisses, gravures, etc. Cette iconographie provient des observations scientifiques, géographiques ou ethnographiques réalisées sur place d'après nature, ou réalisées «après coup " d'après des croquis ou des descriptions textuelles. En effet, les artistes et/ ou scientifiques qui prennent part aux missions dessinent des paysages et portraits souvent destinés à la presse européenne dont les lecteurs sont pour beaucoup fascinés par les découvertes de l'époque et les héros qui en sont à l'origine (Berenson 2010). Par exemple, le peintre franc-comtois Edmond Laethier accompagne Savorgnan de Brazza lors de sa remontée du fleuve Ogooué. Ses dessins sont publiés dès 1887 dans l'hebdomadaire français à large diffusion Le Tour du monde ${ }^{\mathbf{1 4}}$. Jacques Savorgnan de Brazza, docteur ès sciences naturelles, rejoint son frère aîné lors de la troisième expédition et exécute de nombreuses esquisses gravées à son retour en France. D'autres œuvres sont celles d'artistes ne s'étant jamais rendus sur place, à l'instar du peintre et aquafortiste suisse Evert Van Muyden ou de l'illustrateur français Édouard Riou.

Parfois considérée pour sa valeur esthétique, cette iconographie coIoniale est perçue comme constitutive de la production d'un savoir sur cette période. La grande diversité de ses lieux de conservation en France traduit d'ailleurs une certaine confusion: s'agit-il d'objets d'art ou de documents scientifiques? Ainsi, plusieurs images ont rejoint les musées (comme le musée national des Arts africains et océaniens puis le musée du quai Branly ${ }^{\mathbf{1 5}}$, le Muséum national d'histoire naturelle ou encore le musée national de la Marine), tandis que d'autres ont trouvé leur place dans des fonds d'archives
10. Selon l'expression exacte contenue dans le traité du 10 septembre 1880.

11. Missionné par Léopold II, l'explorateur britannique Henry Morton Stanley - le « double diabolique " (Bernault 2010a: 776) de Savorgnan de Brazza - se hâte de fonder Léopoldville (actuelle Kinshasa) en 1884.

12. Voir entre autres Coquery-Vidrovitch (dir.) 1969; Coquery-Vidrovitch 2013 [1972] ; Brunschwig 1972 ; Dion 2007.

13. Parmi ces documents appartenant à un collectionneur parisien, on retrouve par exemple l'original de la lettre de nomination du sergent Malamine et une copie originale du traité conclu entre Makoko et Savorgnan de Brazza. Leur dépôt officiel a été réalisé en 2015 en présence de l'ambassadeur de la République du Congo en France de l'époque, Henri Lopes.
14. "Voyages dans l'Ouest africain de M. Savorgnan de Brazza ", Le Tour du Monde, $2^{\mathrm{e}}$ semestre 1887 et $2^{\mathrm{e}}$ semestre 1888 .

15. Trois carnets de croquis d'Evert Van Muyden sont par exemple désormais conservés au musée du quai Branly.

\author{
double-page \\ suivante \\ fig. 4 \\ Baudouin Mouanda, \\ Fresque du Mémorial \\ Savorgnan de Brazza, \\ Brazzaville, 2016 \\ (c) Baudouin Mouanda
}




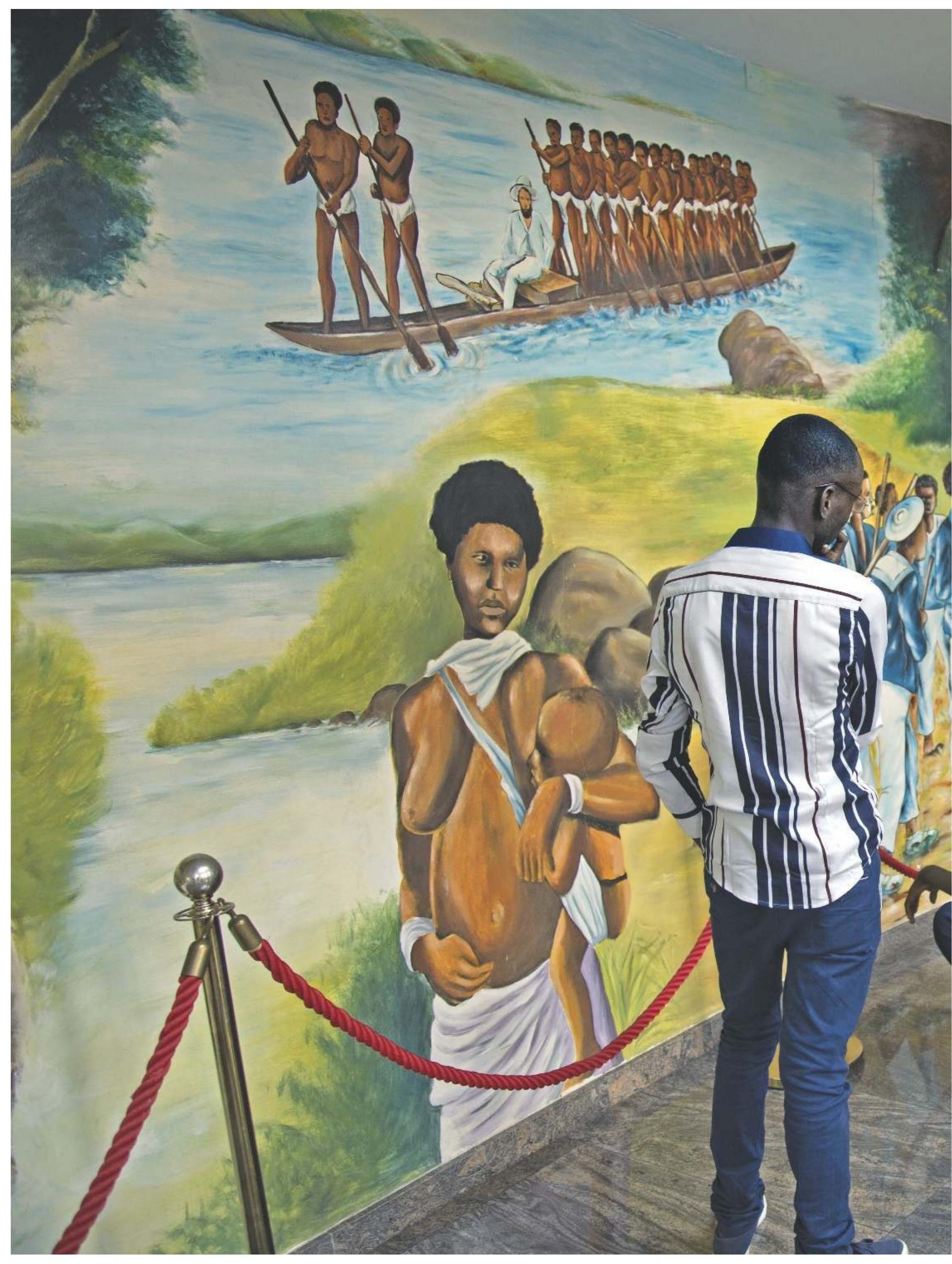


16. La statue en pied installée à l'entrée du mémorial accentue cette image de pacifiste et de libérateur. Inspirée d'un cliché de Nadar, elle représente Savorgnan de Brazza humblement vêtu, tel un apôtre de la mission civilisatrice française. privées et publiques (comme les Archives nationales d'outre-mer, la Bibliothèque nationale de France ou le Centre historique des Archives nationales à Paris).

Voici quelques exemples de réactivations d'archives en cascade. Nous nous situons durant la seconde exploration. Se voyant refuser l'accueil dans un village, un des hommes de Savorgnan de Brazza se fait menaçant et décharge son fusil contre un arbre. II reçoit en retour une balle au poignet. Savorgnan de Brazza explique:

En vain pour l'arrêter j'arrache à un de mes Sénégalais le fusil qu'il vient d'enlever à un naturel et je le remets au chef. Celui-ci le prend, me vise, me manque. Les balles sifflent de tous les côtés et nous comptons six blessés avant de pouvoir nous abriter et battre en retraite. (Ney 1984 [1887]: 183)

En illustrant dans l'enceinte du mémorial cet épisode haletant à partir d'une gravure d'Evert Van Muyden approximativement datée de la fin du $X I X^{e}$ siècle, les peintres de l'École de Poto-Poto opèrent une artification. Ils se font également les porte-parole du célèbre statut de conquérant pacifique de Brazza, celui-ci n'ayant usé qu'a minima de la violence durant ses missions ${ }^{16}$.

Autre exemple d'un discours colonial mis en image, cet extrait d'une lettre de Jacques Savorgnan de Brazza datant de 1883:

Ce matin, j'étais en train de dessiner près de notre campement, et j'avais pour voisin un bel enfant qui me regardait faire; de temps en temps il touchait mon papier, y laissant des marques noires, puis touchait mon crayon, ma pipe, mes habits et me regardait avec ses grands yeux sans bouger et sans avoir peur. (Ibid.: 278)

Là encore, les artistes de Poto-Poto ont reproduit quasiment trait pour trait une gravure d'Evert Van Muyden relatant ces souvenirs. En recopiant le motif d'un homme et d'un enfant de trois quarts dos, accroupis l'un à côté de l'autre, les artistes s'inspirent non seulement du style mais aussi du discours qui sous-tend ce type d'images à la fin du XIX siècle. De la même manière, la scène de la fresque représentant deux hommes creusant dans le sol lors d'une réception solennelle chez le roi Makoko provient d'une gravure réalisée par le Français Fortuné-Louis Méaulle, elle-même issue de la description suivante de Pierre Savorgnan de Brazza:

Le traité étant signé le roi et les chefs mirent un peu de terre dans une boîte. En me la présentant, le grand féticheur me dit: «Prends cette terre et porte-la au grand chef des Blancs; elle lui rappellera que nous lui appartenons. » (Ibid.: 161-162)

La fresque du mémorial constitue une mise bout à bout de ce type de descriptions et d'images d'archives datant de l'époque coloniale. Savorgnan de Brazza y est représenté comme plus habile à la chasse au buffle que ses acolytes noirs puisqu'il manie dextrement le fusil, plus sage car il règle 


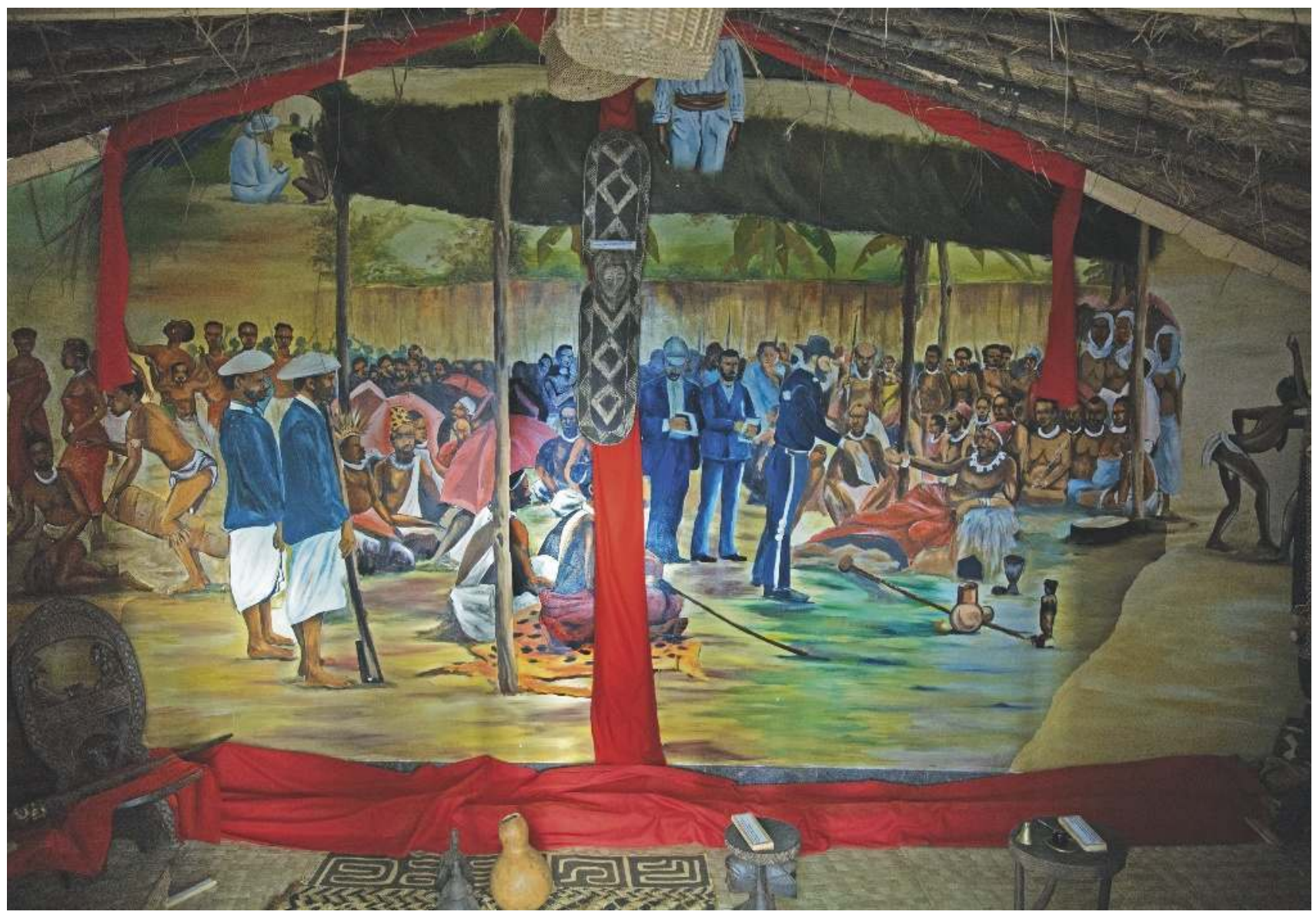

fig. 5

Baudouin Mouanda, Détail de la fresque du Mémorial

Savorgnan de Brazza.

Brazzaville, 2016

(c) Baudouin Mouanda.

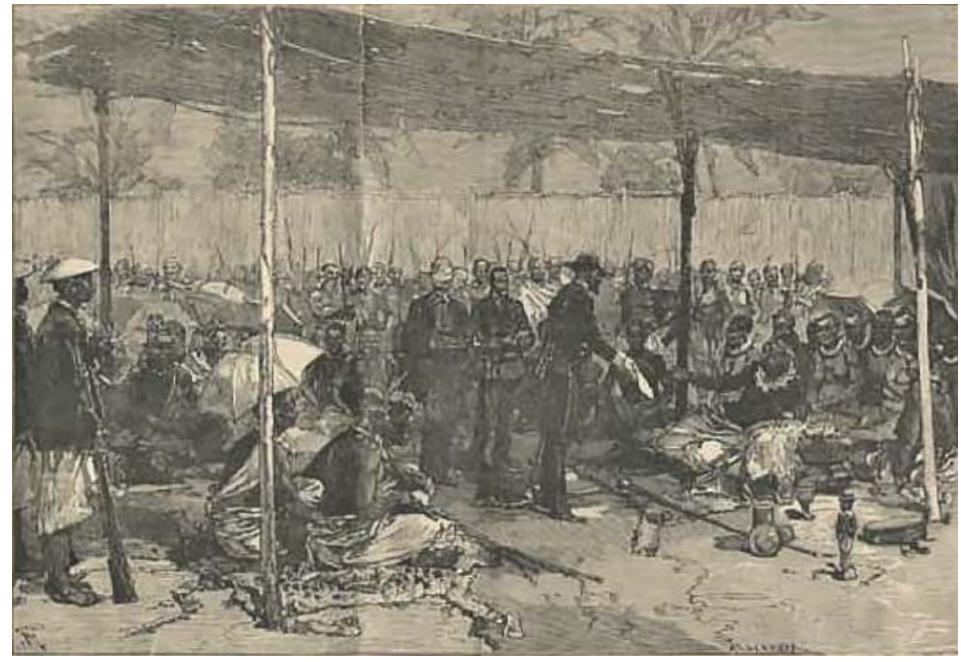

fig. 6

Lithographie représentant

Pierre Savorgnan de

Brazza remettant le traité d'alliance au Makoko (chef coutumier) des Batéké, Iloo ler, le 10 septembre 1880, La République illustrée, 1880. 
17. Les treize peintres réunis, élèves et professeurs de l'école, sont Ngampio, Mpo-Gerly, Mayoulou, Dihoulou, Bokouloumba, Nzimmy, Bogoualanga, Mahoungou, Agnagna, Ngaloutsou, Iloki, Opou et Kimbembe. Outre le fruit de la vente de leurs toiles (dont une partie de la somme est reversée au fonctionnement de l'école), ceux-ci exercent pour la plupart un autre métier (le plus souvent professeur particulier d'art plastique) pour s'assurer une rémunération plus stable. Leur clientèle est composée de personnes issues de la classe aisée, mais surtout d'expatriés désireux de rapporter dans leurs bagages un souvenir typique de la République du Congo.
18. Je parle à ce propos de "mythe de fondation", élaboré au fil des ans et des publications, qui appelle à une relecture critique. En effet, les éléments sémantiques qui le constituent - la rencontre entre un Blanc et un Noir, précisément entre un maître et son domestique, un « coup de foudre " esthétique, un talent africain révélé au grand jour, une interversion de pratique plastique, la fondation d'une école qui en résulte - sont présents dans plusieurs récits antérieurs fondamentaux de l'histoire de l'art africain. L'histoire de l'École de Poto-Poto repose ainsi sur un mythe partagé à l'époque selon lequel il existait une forme d'art de «bons sauvages » qu'il convenait d'encourager et de valoriser. Voir Greani 2009 et Greani 2012.

19. Entretien avec Marcel Gotène (peintre le plus reconnu sur la scène artistique locale, décédé en 2013), Brazzaville, octobre 2010 .

20. D'après Lods luimême, la seule exception concédée, un jour où il présente des peintures religieuses de Raphaël à une élève, s'inscrit dans un cadre strictement expérimental: "Devant cette jeune fille de PotoPoto, apparemment ravie par les images roses et bleues de la bondieuserie, je montrai, pour préparer son "décrassage", des masques bakouele et mpongwé puis les plus belles peintures religieuses de Raphaël. » (Lods 1959: 329)

21. À la demande des autorités religieuses catholiques brazzavilloises, il participe même à la réalisation d'une fresque au sein de la basilique Sainte-Anne à Brazzaville, représentant sainte Anne et Marie à bord d'une pirogue conduite par des anges. les conflits et incite à ne pas utiliser d'armes à feu, etc. Nous nous situons donc dans un traitement non critique d'une histoire faite, écrite et dessinée par les colonisateurs occidentaux; situation qui, pour être mieux comprise, nécessite de se pencher sur l'histoire de l'école d'art la plus célèbre du pays.

\section{Faire émerger l'inspiration à Poto-Poto}

La fresque du mémorial est une commande de l'État passée auprès d'un collectif de peintres de Poto-Poto ${ }^{17}$. Afin de comprendre pourquoi ces artistes ont été mobilisés et dans quelle mesure le regard qu'ils posent sur l'époque coloniale s'inscrit pour ainsi dire dans les "gènes" de l'école, il importe de revenir sur la genèse et la transformation progressive de celle-ci depuis la période précoloniale jusqu’à nos jours.

En 1951, Pierre Lods, officier de marine français nouvellement établi à Brazzaville, découvre fortuitement le talent de l'un de ses domestiques, Félix Ossali. Son éblouissement face à la peinture, sur l'une de ses cartes navales, de motifs «indigènes" (des oiseaux bleus) habituellement présents sur les murs des cases de village le décide à fonder un centre d'art ${ }^{18}$. Ce centre est installé dans l'actuel quartier Moungali, ancienne zone boueuse de la capitale (potopóto signifiant «boue» en lingala). Lods se charge de fournir le matériel qu'il juge nécessaire (gouache, toiles, pinceaux, etc.) et ne prodigue qu'un seul conseil: "Tu imagines tout ce qui te vient à la tête et tu peins, comme ça, sans modèle ${ }^{19}$ " - «modèle» devant avant tout être entendu au sens de modèle de peinture occidentale. Sa méthode de travail se base sur les postulats suivants: primo, ses jeunes élèves seraient préservés depuis toujours de tout contact avec les différentes formes d'art existantes dans le monde; secundo, leur formation au sein du centre favoriserait cet isolement; tertio, il existerait une énergie créatrice "purement " africaine. Or les artistes ont pour la plupart grandi à Brazzaville, grande ville en pleine ébullition à la veille de l'indépendance. Fréquentant par exemple les barsdancings comme Chez Faignond, ils ne perpétuent en rien un mode de vie "typique». Lods entend couper ses protégés de l'art occidental ${ }^{20}$, or son propre penchant pour l'art - il est peintre amateur ${ }^{21}$ - constitue un pont entre ses élèves et l'art occidental. Lods avait d'ailleurs pris conscience de la difficulté à faire émerger une prétendue "africanité profonde " de ces artistes citadins:

Pour alimenter l'inspiration des peintres, je les entoure d'objets africains traditionnels, dans le jardin d'une grande variété de plantes, j'organise des fêtes. Nous lisons quelquefois des légendes africaines, des proverbes, des poèmes qui ont une correspondance pour moi avec le monde noir ou qui participent des mêmes valeurs (Senghor, Césaire, Saint-John Perse, Michaud, Prévert...). (Lods 1959: 329)

Objets d'art premier, décor tropical ou lecture de récits portant sur l'Afrique: la nécessité d'intégrer ces «exhausteurs» de primitivisme ébranle I'hypothèse initiale de Lods de l'existence d'un artiste typique par nature.

Peu à peu, un style propre émerge et prend le nom singulier de «Mickey». Aisément reconnaissables, les œuvres représentatives de ce 
genre pictural sont figuratives, constituées de petites silhouettes aux formes schématisées généralement peintes en noir sur un fond uni de couleur vive. Ces figures sont rapidement associées par les observateurs extérieurs aux personnages des dessins animés de Walt Disney ${ }^{22}$. Ce style rencontre un réel succès sur le marché de l'art. II influence profondément la production artisanale locale et fait toujours la réputation de l'école à l'heure actuelle. Outre le style «Mickey », l'art de Poto-Poto tend à rassembler des œuvres véhiculant une imagerie stéréotypée de l'Afrique (faune et flore tropicale, joueurs de tam-tam, danseurs, etc.) excluant systématiquement toute référence à la modernité.

Durant les années 1950, une autre personnalité investit le centre d'art de Poto-Poto. II s'agit de l'écrivain et critique d'art allemand Rolf Italiaander. Celui-ci rapporte d'Europe des plaques de cuivre qu'il distribue aux élèves ainsi que des modèles de gravures réalisées par des artistes européens ${ }^{\mathbf{2 3}}$. Au même moment ont donc coexisté deux visions distinctes au sein du centre d'art de Poto-Poto: l'une proscrivant officiellement l'influence des modèles occidentaux, l'autre s'appuyant au contraire sur ceux-ci. Ce phénomène relativise, voire contredit, l'idée répandue chez les différents commentateurs de l'art de Poto-Poto selon laquelle les artistes étaient «coupés du monde» sous l'influence de Lods. Italiaander précise toutefois:

Pour tout le reste, je ne les influençais pas. De là, les thèmes qui leur venaient à l'esprit. De là, tous ces danseurs, tous ces animaux, tous ces démons qui apparaissent dans leurs gravures, et aussi les scènes de la vie à Poto-Poto, tels qu'ils les voient. Mais aucune des maisons à l'européenne, des voitures, des motos, des bicyclettes qu'ils rêvaient pourtant de posséder: en général, aucun symbole du $x^{\mathrm{e}}$ siècle. (Italiaander 1960: 230)

Voici un motif d'interrogation pertinent: pourquoi ces artistes, apparemment libres de choisir leurs propres thématiques, se restreignaient-ils volontairement et systématiquement à une vision traditionnelle de l'Afrique, excluant tout indice de modernité? Italiaander propose l'explication suivante: «Il faut peut-être chercher la cause du phénomène en question dans un sorte de passivité ou d'indolence. Une fois qu'ils ont appris une chose, ils s'y fixent et recommencent indéfiniment ce qu'ils ont réussi. » (Ibid. : 230-231) Ces mots prononcés lors d'une conférence de la Société des africanistes sont le produit d'un lieu et d'une époque. Près de cinquante ans plus tard, d'autres raisons peuvent être avancées concernant l'étonnante uniformisation thématique des artistes qui court jusqu'à nos jours. Les artistes réunis par Lods ne sont pas des enfants de familles fortunées souhaitant profiter de leur temps libre pour s'initier à l'art. Souvent, ils ne savent ni lire ni écrire. Certains n'ont pas d'emploi; la plupart exercent des métiers leur procurant le minimum pour survivre. Et voici que, brusquement, l'un d'entre eux voit son statut social se métamorphoser grâce à l'émerveillement de son patron blanc devant une reproduction des motifs peints sur les murs de cases. Il devient alors un homme respecté, comme l'explique Sylvestre Mangouandza, peintre-professeur membre de l'École de Poto-Poto, lors d'un entretien en janvier 2012 à Brazzaville:
22. Selon les versions, cette analogie provient soit de spectateurs congolais, soit d'un journaliste français qui aurait traduit le mot lingala «mike» (pluriel de "moke") signifiant "petits" ou «minces " par un homonyme approximatif, «Mickey», et aurait diffusé l'expression.

23. Lods lui-même ne souffle mot de cette expérience lors du second Congrès des écrivains et artistes noirs. 


\title{
LE MONDE ILLISTRE F $^{3123}$ JoonNAL HEBD OMADARAB
}

\begin{abstract}
ABOHNEMENTS POUR PARIS ET LES DEPARTEMENTS

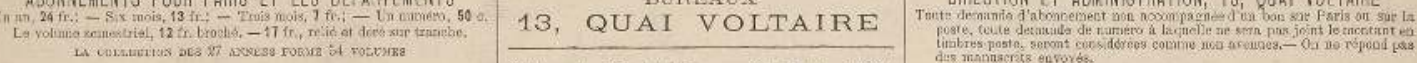

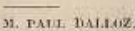

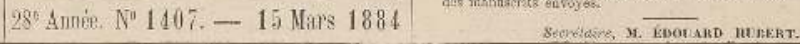

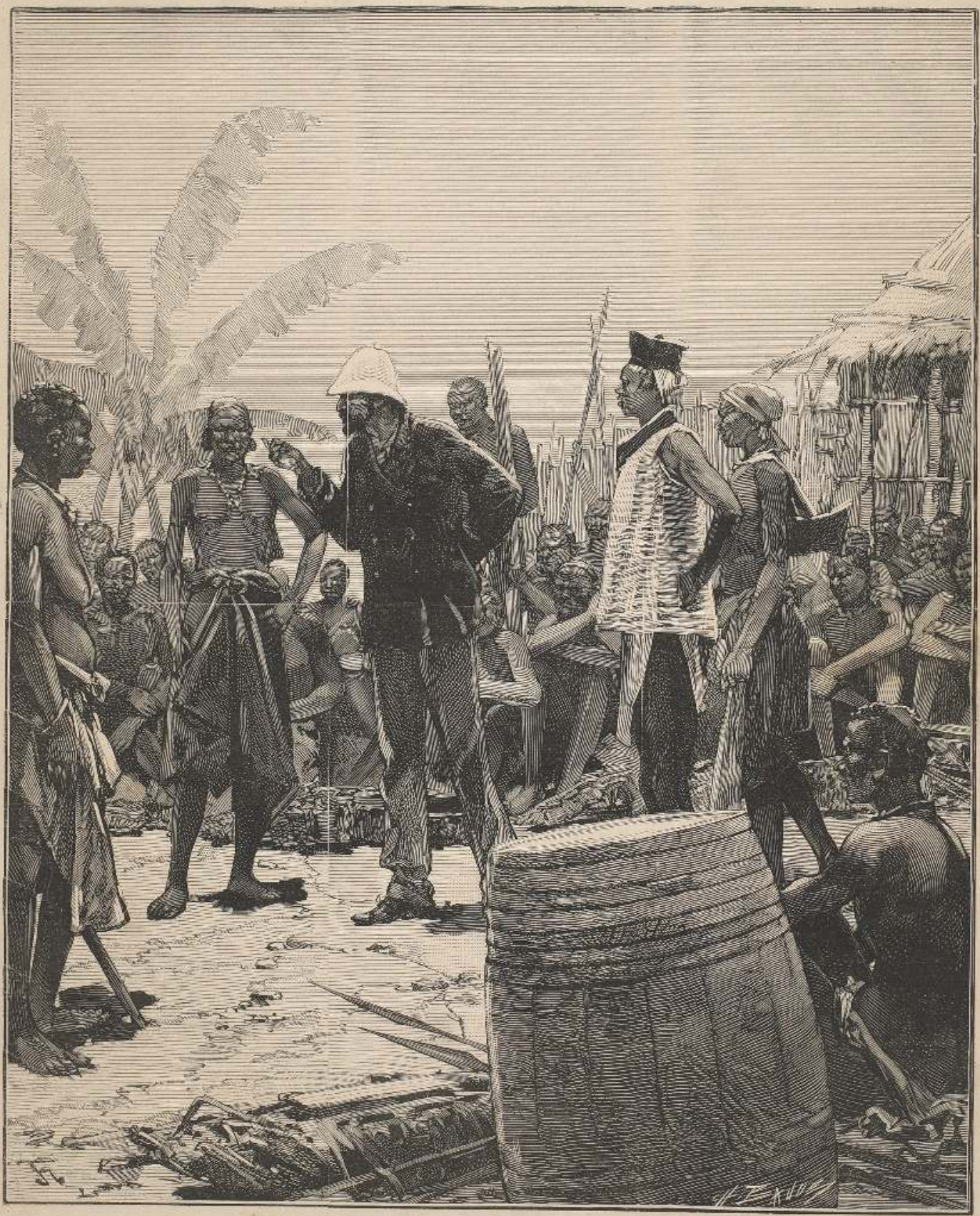

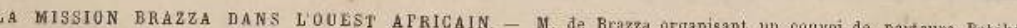
dricaine, le 14 decembre 1804 - 
Être à côté de l'homme blanc, c'était un luxe à l'époque. Ondongo, Ossali, ils étaient avec Pierre [Lods], alors ils avaient des culottes kaki, avec une belle ceinture, des babouches. Quand ils partaient à Kinshasa, les gens étaient impressionnés. Ils restaient à dix mètres!

On peut penser que l'engouement des artistes de Poto-Poto pour des thématiques "authentiques", développées au sein d'œuvres originales ou de reproductions de style "Mickey", relève en partie d'une stratégie sociale et économique. En effet, il semble tentant de reconstituer à l'attention des "protecteurs blancs" (Lods, Italiaander ou les clients d'une manière générale) une Afrique originelle qui semble les fasciner, surtout lorsque cette activité favorise sa propre propulsion vers des positions sociales nettement supérieures à celles occupées jusque-là. Toutefois, de nombreux artistes interrogés, actuels ou anciens membres de l'école, analysent cette situation différemment: l'intérêt des Occidentaux pour les artistes pionniers est une marque de reconnaissance du "génie" de ces derniers. Loin d'avoir été conditionnés par les regards extérieurs, ils ont élaboré un style et un vocabulaire iconographique originaux que les nouvelles générations s'efforcent de perpétuer à l'heure actuelle. Les peintres de Poto-Poto sont ainsi devenus des ambassadeurs « interchangeables ${ }^{\mathbf{2 4}}$ » de la culture congolaise à travers le monde, signant «PPP» pour «Peintres de Poto-Poto».

Désormais, parmi les objets qui trônent dans l'école, on trouve toutes sortes d'ouvrages d'art laissés en cadeau par les visiteurs. L'un d'eux a été à l'origine de la fresque du mémorial: intitulé Una vita per l'Africa, il a été rédigé et offert avant sa parution officielle en 2006 par l'une des descendantes de Savorgnan de Brazza, Idanna Pucci. Ce livre contient toutes les reproductions de l'imagerie coloniale dont se sont inspirés les artistes pour la fresque ${ }^{25}$. Ce don s'inscrit dans le cadre plus général d'échanges directs avec cette famille. Ainsi, à la suite d'une visite en 2005, les membres de l'école ont reçu de sa part du matériel introuvable sur place depuis la guerre civile (essentiellement de la peinture acrylique) ayant permis la confection d'un polyptique de douze tableaux honorant la mémoire de Savorgnan de Brazza. La confection de ce polyptique s'inspire d'une perception «lodsienne» de l'art, comme le relate Idanna Pucci:

Naturellement, les artistes me posèrent une infinité de questions sur l'éventuel sujet de l'œuvre: je leurs répondais qu'ils étaient libres de faire ce qu'ils souhaitaient à condition de respecter les principes originaires de leur école. Car j'avais remarqué que, récemment, certains d'entre eux avaient compromis leur inspiration pour satisfaire le goût des clients étrangers. Mais, conditionnés par la perspective de pouvoir enfin utiliser leurs talents spontanés, je ne voyais autour de moi que des visages souriants et des yeux resplendissants de lumière. (Pucci 2006)

La commande de cette vaste œuvre désormais installée au Musée national du Cameroun laissait donc une marge de créativité aux artistes, ce qui ne fut pas le cas avec la fresque du mémorial, directement influencée par le don des descendants d'une iconographie tenant lieu de modèle. Les conditions de la commande, non plus passée par un mécène étranger mais par
24. Il est toutefois possible de distinguer «la pâte" de chaque artiste (traitement des couleurs ou de la composition, thématiques spécifiques, etc.) s'efforçant ainsi de se distinguer tout en s'inscrivant dans la mouvance de l'école.

25. Dès 1887 , une première compilation de l'iconographie disponible est opérée à l'occasion de la parution de l'ouvrage Conférences et lettres de P. Savorgnan de Brazza sur ses trois explorations dans l'Ouest africain, de 1875 à 1886 (Ney 1984 [1887]).

\section{ci-contre}

fig. 7

La mission Brazza dans

I'Ouest africain. Un convoi de Batéké à Franceville, Le Monde illustré, 15 mars 1884. Coll. ANOM.

\section{double page} suivante

fig. 8

Baudouin Mouanda, Exposition devant la fresque du Mémorial Savorgnan de Brazza, Brazzaville, 2016 () Baudouin Mouanda. 


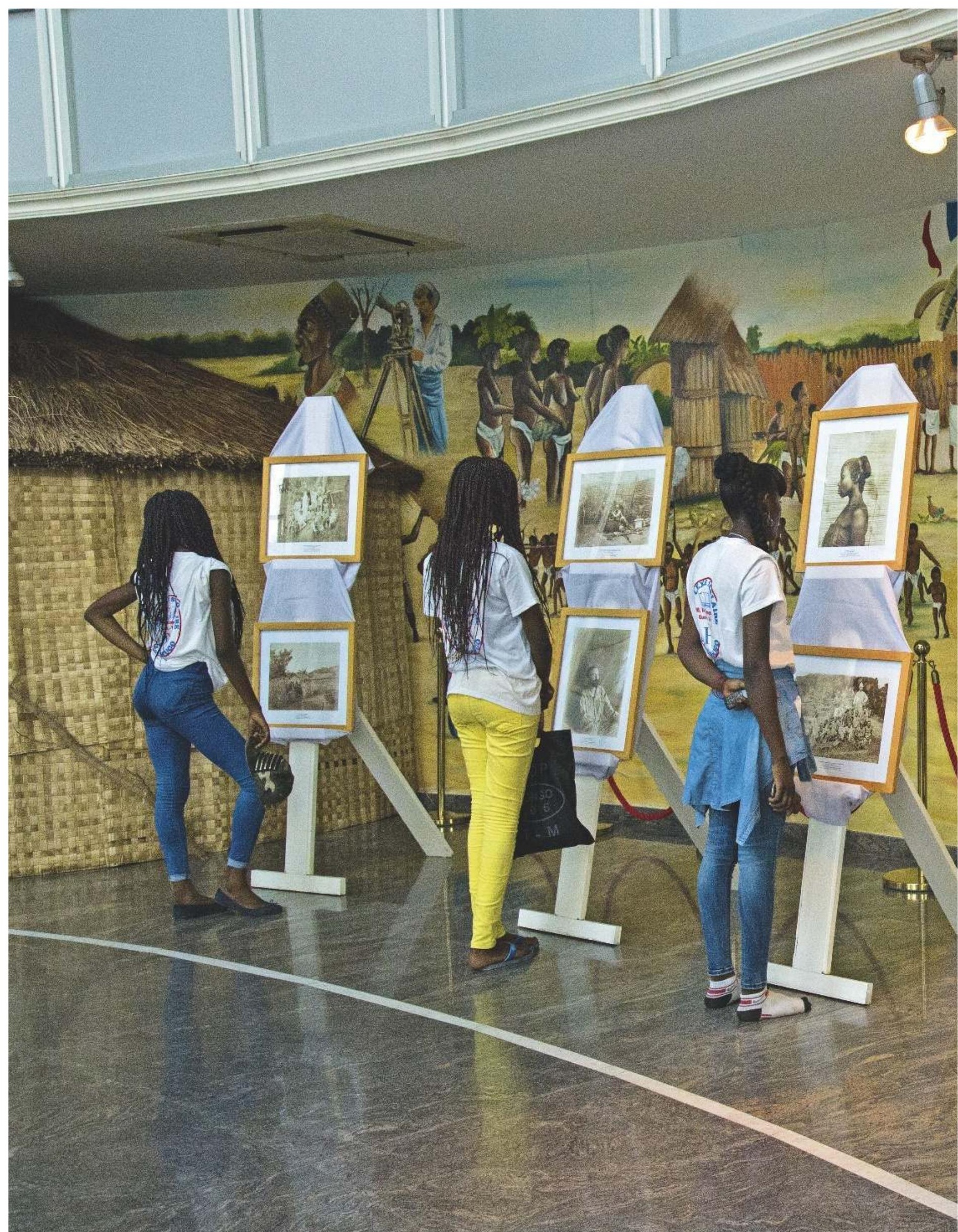




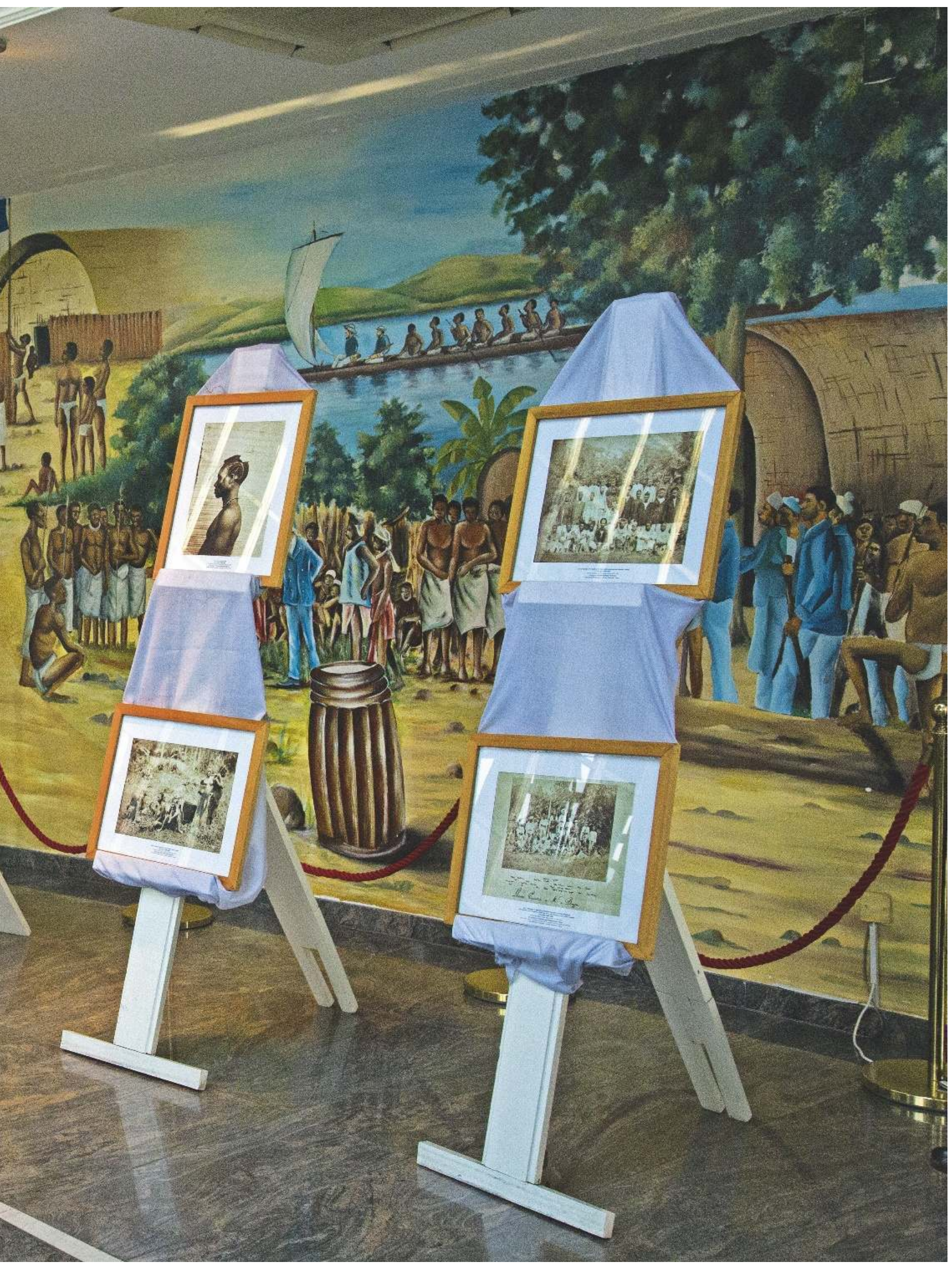


26. En effet, certains descendants ont appelé de leurs vœux le retour des restes mortuaires de leur ancêtre en Italie, estimant que le gouvernement congolais n'avait pas tenu ses promesses (concernant, par exemple, le bitumage de la piste conduisant au dispensaire de Mbé ou l'entretien du lycée Savorgnan-de-Brazza)

27. L'œuvre n'a pas été détruite, certes, mais n'a pas non plus été entretenue; ce qui, à plus ou moins longue échéance, revient au même.
l'État, y sont pour beaucoup. La fresque a été envisagée avant tout comme une œuvre historique décorative plutôt que comme une œuvre d'art contemporain. Par ailleurs, en dépit de son statut d'institution privée, l'école bénéficie d'une subvention de l'État qui assigne ses membres au rang d'artisans - plutôt que d'artistes - officiels.

Si de nos jours les relations entre l'école et la famille Brazza sont plus incertaines ${ }^{26}$, les chemises de couleurs vives flanquées du portrait de Savorgnan de Brazza que portent certains de ses membres témoignent pourtant de la prégnance de ces liens.

\section{Deux discours pour une même archive}

Réalisée par quatre peintres congolais sous la supervision d'un artiste italien, la Fresque de l'Afrique constitue une commande de la direction générale des Affaires culturelles en 1970. II s'agit de la première commande culturelle d'envergure émanant de la sphère politique. CEuvre révélatrice de l'iconographie du second parti unique, elle présente l'intérêt d'être toujours en place dans le centre-ville de la capitale ${ }^{27}$. Afin de saisir la «leçon d'histoire» de ce monument, il convient de l'observer en un mouvement ascendant. L'histoire est représentée de manière fragmentée de bas en haut, depuis la période précoloniale jusqu'à l'avènement du socialisme scientifique. Au niveau de la bande inférieure, une scène présente la vie des Africains avant l'arrivée des Blancs. Les hommes s'emploient à la pêche, à la chasse et à la culture des champs tandis que le chef de village, assis sur un trône et respecté de tous, veille sur eux. Un baobab regorgeant de pains de singe symbolise une ère d'abondance. Le caractère guerrier ou chasseur de cette société ancestrale est discrètement signifié par une file d'hommes armés de lances et de boucliers. II s'agit d'une vision idéalisée du passé précolonial présenté comme basé sur l'autosuffisance et la cohérence de son organisation sociale, destinée à trancher avec le cours ultérieur de l'histoire représenté plus haut. En effet, les deux colonnes de l'arche dispensent des repères mémoriels en rapport avec la pénétration coloniale dans l'économie, la société et la pensée. Au sommet, l'avènement du socialisme scientifique est présenté comme un nouveau stade historique caractérisé par le progrès, comme en témoignent des usines empanachées de fumée ou un paquebot géant, respectivement synonymes de labeur prolétarien et d'ouverture sur le monde. II s'agit de montrer la rupture essentielle entre un stade archaïque et une société moderne industrialisée. Le passé mythifié vient souligner l'amélioration des conditions de vie de la population grâce aux équipements modernes.

L'arche entend dénoncer les expéditions militaires occidentales et l'évangélisation forcée. Une plaque commémorative peinte rappelle ainsi que cinquante-cinq millions d'Africains ont été déportés en esclavage. Les colons, reconnaissables à leurs vêtements de brousse beiges et leurs casques coloniaux, sont dépeints comme violents, astreignant les Africains aux travaux forcés, et dédaigneux, leur goût pour le tipoye (chaise à porteurs) en témoignant. Des prêtres et des religieuses sont représentés sur plusieurs carreaux comme des êtres à la fois cruels - prêchant devant un amas de cadavres - et ridicules - prenant la fuite à toutes jambes, leurs croix sous 
le bras, pour échapper à la vindicte populaire. La valeur des indices iconographiques religieux (croix latine, soutane, procession, etc.) est ici renversée afin de mettre au jour le rôle néfaste de l'expansion du christianisme en Afrique.

Trente-six ans plus tard, dans la fresque du mémorial, la période précoloniale du début du protectorat français ne correspond plus à une tragédie annonçant la mort de milliers d'Africains, mais à un événement bénéfique pour les populations locales. Si la représentation de colons avachis sur des tipoyes cherchait à éveiller chez le spectateur un sentiment d'injustice, ici, au contraire, la position confortablement assise de Savorgnan de Brazza dans une pirogue menée à la pagaie par pas moins de dix-huit Africains debout (deux devant et seize derrière) est présentée comme légitime. Les tensions permanentes entre Noirs et Blancs relatées dans la fresque de 1970 disparaissent pour laisser place à un message d'alliance. C'est d'ailleurs un Africain qui hisse avec fierté le drapeau français à l'entrée de son village, applaudi par la foule.

Étant entendu que la fresque du mémorial offre la vision du double opposé du Blanc proposée dans la Fresque de l'Afrique - pacifiste dans un cas, sanguinaire dans l'autre $\mathbf{2 8}^{\mathbf{2}}$, examinons à présent l'imaginaire qui fonde la représentation des Congolais dans les deux œuvres. Sur les colonnes de l'arche, hommes et femmes luttent courageusement contre l'oppression extérieure, souvent au prix de leur vie. Au niveau du fronton, ils deviennent des figures héroïques. Affranchis du joug colonial passé, ils se relèvent et se tournent vers un avenir marqué du sceau socialiste. À l'inverse, dans le mémorial, la population congolaise reçoit avec gratitude l'hôte étranger. Ignorante et docile, simplement vêtue de pagnes ou de culottes blanches, elle semble retrouver les attributs d'une peuplade «primitive».

La représentation de la femme diffère tout particulièrement d'une fresque à l'autre. Dans la Fresque de l'Afrique, les femmes sont données à voir à plusieurs reprises en meneuses d'un mouvement révolutionnaire composé d'hommes, ce qui suggère que les hommes et les femmes jouent un rôle à part égale dans la révolution. L'une d'elles, mère musculeuse aux mains libres (elle porte son enfant sur son dos, comme il est d'usage), est représentée comme une militante anonyme du parti et est visiblement donnée en modèle à suivre par toutes les Congolaises. Cette femme émancipée n'a rien à voir avec celle qui, dans l'autre fresque, porte son enfant contre sa poitrine découverte et semble se consacrer au seul rôle de mère et de femme au foyer.

On trouve par ailleurs dans les deux fresques la représentation d'un même épisode historique. II s'agit de la remise du traité conclu en 1880 entre Savorgnan de Brazza et Makoko au cours de la troisième mission. Voici comment Savorgnan de Brazza rend compte de l'événement:

Est-il indispensable de dire que le cérémonial de réception n'avait point tout à fait la rigoureuse correction d'étiquette exigée en pareil cas dans nos pays? [...] Makoko trônait sur ses peaux de lion, négligemment
28. Au sujet de l'image de l'homme blanc dans l'iconographie coloniale, voir Blanchard et Böetsche 2013. 


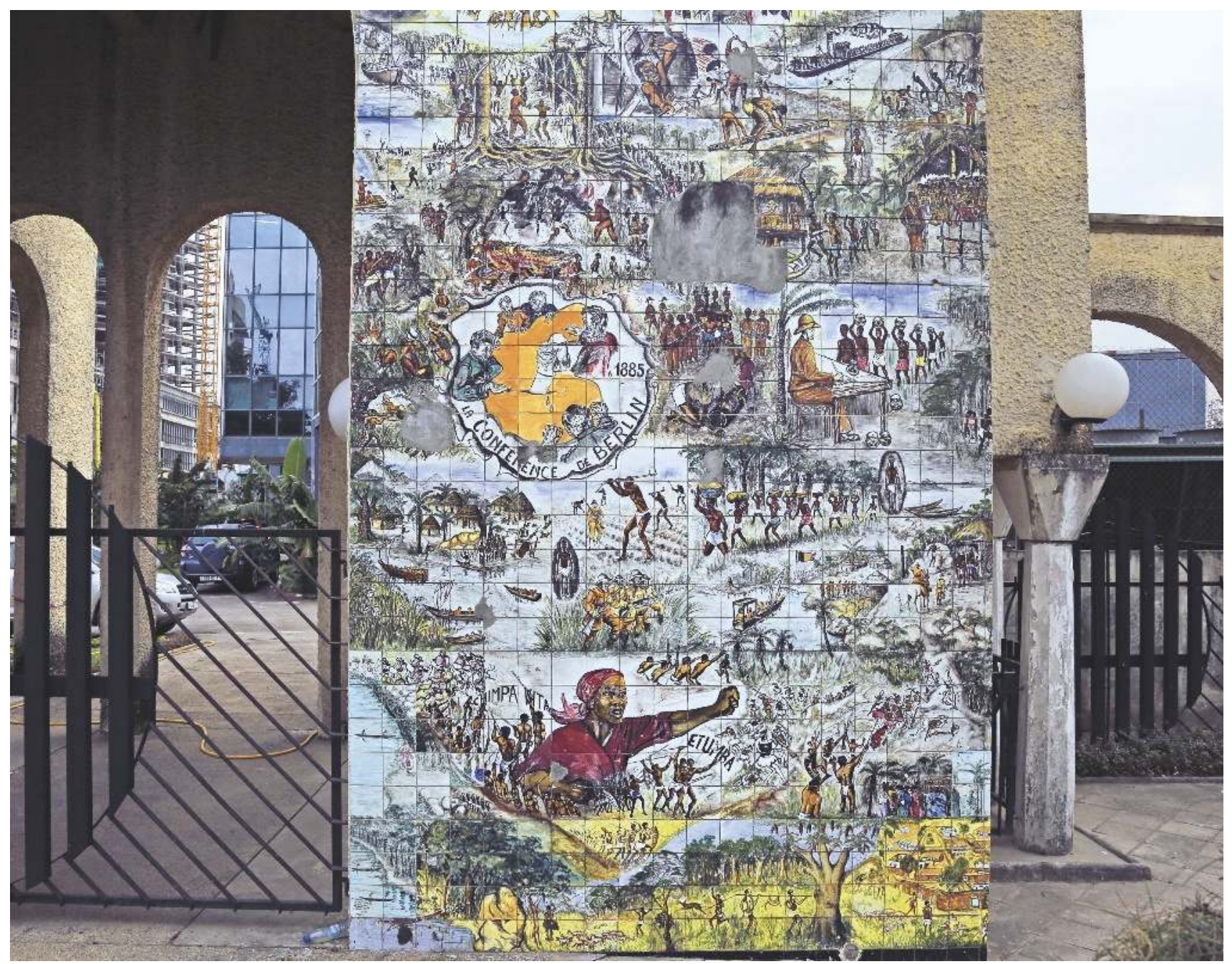


accoudé sur des coussins, entouré de ses femmes et de ses favoris. [...] Tous se déclarèrent, comme autrefois, heureux et fiers d'être placés sous la protection de notre drapeau et le jurèrent sur les fétiches et par les mânes de leurs pères. À mon tour je rappelai le passé en quelques mots. Mes hommes présentaient les armes, on sonna aux champs et je fis à Makoko la remise des traités au nom de la France. Procès-verbal de la cérémonie fut dressé et signé [...]. On se dit gaiement au revoir. (Ney 1984 [1887]: 232)

Cet épisode crucial est commémoré de façon quasiment identique dans les deux fresques par la mise en scène de Savorgnan de Brazza, de trois quarts dos, présentant une feuille de papier au roi étendu. Hormis quelques détails (par exemple la feuille du traité est tantôt vierge, tantôt signée des noms «Brazza» et «Makoko»), les points de recoupement entre les deux œuvres sont suffisamment nombreux pour attester qu'elles réactivent la même gravure de l'époque coloniale. Si le côté rétro de l'archive en noir et blanc est modernisée par sa colorisation, il s'agit d'une citation exacte du même modèle. Pour autant, la scène véhicule des messages distincts. En 2006, dans la fresque du mémorial, le pacte semble se fonder sur un échange équitable, acclamé par le peuple qui reçoit en échange un drapeau français, symbole de protection dans l'avenir. À l'inverse, en 1970, dans la Fresque de l'Afrique, le traité constitue en quelque sorte un marché de dupes. Le Makoko cède l'exercice de ses privilèges à la France sans avoir bien saisi les implications futures de sa décision. En effet, la scène a pour pendant (sur la colonne droite) un autre moment clé de l'histoire coloniale, la Conférence de Berlin. Les affreuses mains griffues des participants tranchent au poignard dans l'Afrique tandis que perlent deux gouttes de sang. Ainsi, les représentations du pacte et de la conférence dénoncent la domination et la malveillance occidentales qui s'exercent sur le continent africain.

Une même archive coloniale peut donc successivement légitimer ou contester l'entreprise coloniale sur le plan symbolique. Dans les deux cas, les artistes opposent de manière binaire les colonisateurs et les colonisés, les vainqueurs et les vaincus, les Blancs et les Noirs. Ces représentations concurrentes sont liées aux intérêts politiques et au contexte historique de 1970 puis de 2006. Commandes officielles, les deux fresques s'inscrivent dans le cadre de l'État-nation et attestent d'un rapport ambigu à l'époque coloniale et au passé d'une manière générale. L'histoire est fragmentée: découpée, redécoupée et réassemblée pour produire un nouveau discours. Ces deux œuvres éclairent ainsi les usages officiels et populaires de l'art, que ce soit au sein d'une nation fraîchement indépendante ou dans un contexte postcolonial complexe.
Chercheuse associée au IIAC-Lahic nora.greani@gmail.com

\section{ci-contre}

fig. 9

Baudouin Mouanda,

Fresque de l'Afrique,

détail, Brazzaville, 2016

(c) Baudouin Mouanda. 


\section{Atondji-Monmondji, Lecas}

2006 «Pouvoir congolais et révisionnisme post-colonial: Le cas Savorgnan de Brazza» [en ligne], disponible sur: www.congopage.com/Pouvoircongolais-et-revionniste

\section{Bayart, Jean-François}

1981 «Le politique par le bas en Afrique noire. Questions de méthode ", Politique africaine 1 : 53-82.

\section{Bayart, Jean-François, Mbembe, Achille et Toulabor, Comi}

2008 [1981], Le Politique par le bas en Afrique noire: contributions à une problématique de la démocratie. Paris, Karthala.

\section{Bazenguissa-Ganga, Rémy}

2014 "Les "os de la République" ou l'ancestralisation contestée au Congo-Brazzaville", Civilisations 63 $27-44$

\section{Berenson, Edward}

2010 "Le charisme et la construction des héros de l'Empire en Grande-Bretagne et en France. 1880-1914 ", Actes de la recherche en sciences sociales 185: 62-81.

\section{Bernault, Florence}

1996 Démocraties ambigües en Afrique centrale: Congo-Brazzaville, Gabon (1940-1956). Paris, Karthala

2010a «Quelque chose de pourri dans le post-empire. Le fétiche. le corps et la marchandise dans le Mémorial de Brazza au Congo ", Cahiers d'études africaines 198-199-200: 771-797.

2010b «Colonial Bones. The 2006 Burial of Savorgnan de Brazza in the Congo ", African Affairs 436 : 367-390.

\section{Bertrand, Hugues \\ 1975 Le Congo: formation sociale et mode de développement économique. Paris, François Maspero. \\ Blanchard, Pascal et Boëtsch, Gilles}

2013 "La construction du "Blanc" dans l'iconographie coloniale", in Sylvie Laurent et Thierry Leclère (dir.), De quelle couleur sont les blancs? Des "petits Blancs» des colonies au "racisme anti-Blancs». Paris, La Découverte: 128-140.

\section{Boilley, Pierre}

2005 "Loi du 23 février 2005, colonisation, indigènes, victimisations. Évocations binaires, représentations primaires ", Politique africaine 98: 131-140.

\section{Bonhomme, Julien et} Jaoul, Nicolas (dir.)

2010 "Grands hommes vus d'en bas. L'iconographie officielle et ses usages populaires », Gradhiva 11.

\section{Bonnafé, Pierre}

1968 «Une classe d'âge politique: la JMNR de la République du Congo-Brazzaville ", Cahiers d'études africaines 8: 327-368.

\section{Brunschwig, Henri}

1972 Brazza explorateur: les traités Makoko (1880-1882). Paris, Éditions de l'EHESS.

\section{Coquery-Vidrovitch, Catherine (dir.)}

1969 Brazza et la prise de possession du Congo: la mission de l'ouest africain, 1883-1885. Paris, Mouton et Cie.

2013 [1972] Le Congo au temps des grandes compagnies concessionnaires, 1898-1930. Paris, Éditions de l'EHESS.

\section{De Jong, Ferdinand et} Harney, Elisabeth (dir.)

2015 "Art from the Archive", African Arts 48 (2).

\section{Dion, Isabelle}

2007 Pierre Savorgnan de Brazza, au cœur du Congo. Marseille, Images en manœuvres.

\section{Greani, Nora}

$\mathbf{2 0 0 9}$ «Art sous influences. Une approche anthropologique de la créativité contemporaine au Congo-Brazzaville ", thèse de doctorat, École des hautes études en sciences sociales, sous la direction de Jean-Loup Amselle.

2012 "Soixante ans de création à l'École de peinture de Poto Poto (Congo-Brazzaville) ", Cahiers d'études africaines 205: 259-267.

\section{Gruénais, Marc-Éric,} Mouanda Mbambi, Florent et Tonda, Joseph

1995 «Messies, fétiches et lutte de pouvoirs entre les "grands hommes" du Congo démocratique ", Cahiers d'études africaines 137 : 163-193.

\section{Hadjinicolaou, Nicos}

1979 "La Liberté guidant le peuple de Delacroix devant son premier public ", Actes de la recherche en sciences sociales $28: 3-26$.

\section{Heinich, Nathalie et Schapiro, Roberta (dir.)}

2012 De l'artification. Enquêtes sur le passage à l'art. Paris, Éditions de l'EHESS.

\section{Italiaander, Rolf}

1960 «Les graveurs de Poto-Poto. Résumé de la Conférence du professeur Rolf Italiaander (décembre 1960, Société des africanistes)", Journal de la Société des africanistes 30: 229-231.

\section{Latour, Bruno}

2009 Sur le culte moderne des dieux faitiches, suivi de Iconoclash. Paris, Les empêcheurs de penser en rond/La Découverte.

Le Lay, Maëline, Malaquais, Dominique et Siegert, Nadine (dir.)

2015 Archive (re)mix: vues d'Afrique. Rennes, Presses universitaires de Rennes.

\section{Lods, Pierre}

1959 "Les peintres de Poto-Poto", Présence africaine 24-25: 326-330.

\section{Martin-Granel, Nicolas}

2010 “"Abracadabrazza" ou le roman du Mémorial Pierre Savorgnan de Brazza ", Cahiers d'études africaines 197 : 293-307.

\section{Ney, Napoléon (éd.)}

1984 [1887] Conférences et lettres de P. Savorgnan de Brazza sur ses trois explorations dans l'Ouest africain, de 1875 à 1886 . Brazzaville/ Heidelberg, Éditions Bantoues.

\section{Poirier, Léon}

1939 Brazza ou l'Épopée du Congo. Société de production du film Brazza, 98 minutes.

\section{Pucci, Idanna}

2006 Una vita per l'Africa, Pietro Savorgnan di Brazzà, Makoko Iloo I. Florence, Libreria editrice fiorentina.

2006 "Du rêve au tableau " [en ligne], disponible sur: www.fabrizioruggiero.it/ CAMEROON/DU_R\%C3\%8AVE AU_TABLEAU.html (consulté le $1^{\text {er }}$ septembre 2016).

\section{Stoler, Ann Laura}

2010 Along the Archival Grain: epistemic Anxieties and Colonial Common Sense. Princeton/Oxford, Princeton University Press. 


\section{Tonda, Joseph}

2002 La Guérison divine en Afrique centrale (Congo, Gabon). Paris, Karthala.

\section{0 "Le Mausolée Brazza}

corps mystique de l'État congolais ou corps du "négatif" ", Cahiers d'études africaines 198-199-200: 799-821.

\section{Yengo, Patrice}

2006 La Guerre civile du CongoBrazzaville (1993-2002) : chacun aura sa part. Paris, Karthala. page 82 et ci-contre Baudouin Mouanda, Détail de la fresque du Mémorial Savorgnan de Brazza, Brazzaville, 2016 C Baudouin Mouanda.

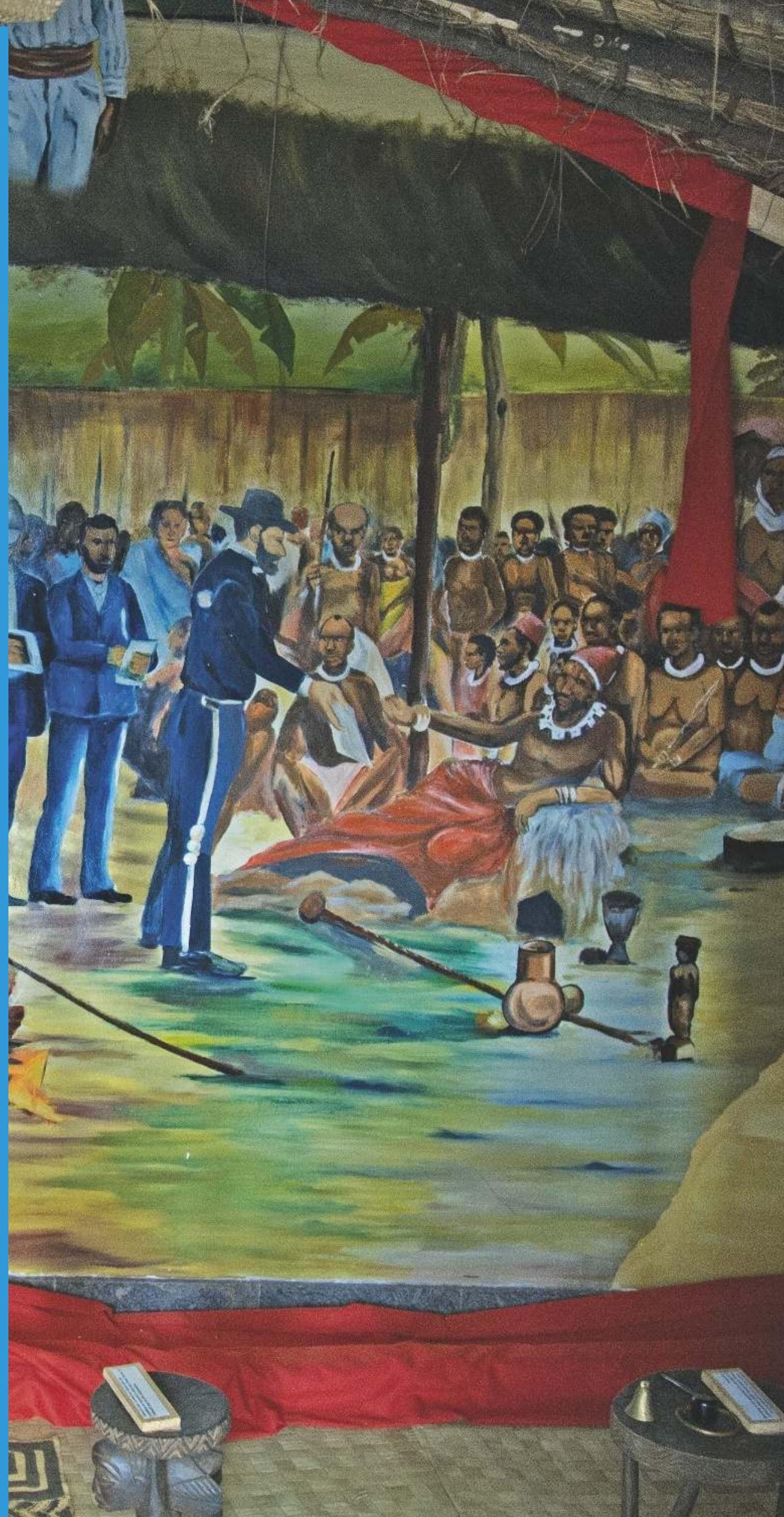

